\title{
Cryo-EM structure determination of small protein complexes with the Volta phase plate
}

\author{
Maryam Khoshouei ${ }^{1}$, Radostin Danev ${ }^{1}$, Mazdak Radjainia ${ }^{2}$, Wolfgang Baumeister ${ }^{1}$ \\ 1 - Department of Molecular Structural Biology, Max Planck Institute of Biochemistry, 82152 Martinsried, Germany. \\ 2 - FEI, 5651 GG Eindhoven, The Netherlands.
}

Phase contrast microscopy has been used in light microscopy for imaging unstained biological material for more than 80 years, opens up new possibilities in structural biology by cryo-electron microscopy (cryoEM). Images of unstained and frozen-hydrated biological material produce very little contrast, since such samples interact weakly with high-energy electrons. The only way to produce contrast in cryo-EM (until recently) was to intentionally defocus the image at the expense of altering high-resolution structural information. Despite the various efforts made in the development of EM phase plates, the technique was not readily applicable for cryo-EM, mainly due to the very short 'life time' of a phase plate and its complicated use.

However, we have developed the new generation of the thin film phase plate at Max-Planck Institute of Biochemistry in Martinsried, Germay, which succeeded in creating a device called the Volta phase plate (VPP) that nowadays is routinely used for data collection in cryo-EM. As a result, it creates contrast for observation of weak phase objects by boosting the low frequency information, without the need of defocusing.

Here, I will show how phase contrast cryo-EM enables single particle analysis of a challenging sample in terms of size that is difficult to solve by any other approach. Because of the high contrast it provides, VPP imaging could moreover become an ideal tool for quick sample screening, initial model building and/or structure determination of small complexes at atomic resolutions. 